Journal of Fluid Mechanics

http://journals.cambridge.org/FLM

Journal of

Additional services for Journal of Fluid Mechanics:

Email alerts: $\underline{\text { Click here }}$

Subscriptions: $\underline{\text { Click here }}$

Commercial reprints: $\underline{\text { Click here }}$

Terms of use : $\underline{\text { Click here }}$

\title{
Lifting multi-blade flows with interaction
}

R. G. A. BOWLES and F. T. SMITH

Journal of Fluid Mechanics / Volume 415 / July 2000, pp 203 - 226

DOI: 10.1017/S0022112000008673, Published online: 08 September 2000

Link to this article: http://journals.cambridge.org/abstract_S0022112000008673

How to cite this article:

R. G. A. BOWLES and F. T. SMITH (2000). Lifting multi-blade flows with interaction. Journal of Fluid Mechanics, 415, pp 203-226 doi:10.1017/S0022112000008673

Request Permissions : $\underline{\text { Click here }}$ 


\title{
Lifting multi-blade flows with interaction
}

\author{
By R. G. A. BOWLES AND F. T. SMITH \\ Mathematics Department, University College London, Gower Street, London WC1E 6BT
}

(Received 7 October 1999 and in revised form 6 March 2000)

Planar flow past multiple successive blades and wakes is studied for nearly aligned configurations with normal non-symmetry inducing lift. The typical blade lies relatively near the centreline of the oncoming wake from the preceding blade. The central motion over a wide parameter range is in condensed periodic boundary layers and wakes with fixed displacement, buried within surrounding incident shear flow. This is accompanied, however, by streamwise jumps in the pressure, velocity and mass flux, across the leading edge of each blade, a new and surprising feature which is supported by the combination of incident shears and a solid surface and which is related to the normal flow through the multi-blade system. The leading-edge jumps are required in order to satisfy the equi-pressure condition at the trailing edge. Computational results include separating flows and show the lift and drag, and these are followed by a short-blade analysis which captures the main flow properties explicitly. The results agree qualitatively with experiments and direct simulations for rotor blade flows. The jump feature also extends for example to a single blade immersed in the relatively large wake of an upstream blade.

\section{Introduction}

Arrangements of multiple successive blades in near alignment with a fluid stream, so that each blade lies in or close to the wake of the previous blade, are involved in numerous practical applications. These include flows past helicopters, other rotorcraft, propellers and turbine blades, and in engines, food mixers, blenders, bean grinders, hover mowers, fans, industrial mixers, as well as for multiple airfoil flaps, slipstreaming, airborne seed travel, flocks of migrating birds, pursuit and landing or take-off delay issues. The characteristic Reynolds number is usually large in all these cases, and the blades or equivalent airfoils tend to be thin.

The applications above have in turn led to numerous flow studies, many of which however focus on properties for an isolated blade. Computations based on an inviscid approximation or on direct numerical simulation at relatively low Reynolds numbers, and some experimental work, are discussed mostly for the helicopter setting by Egolf \& Sparks (1985), Davis \& Chang (1986), Strawn \& Caradonna (1986), Lorber (1990), Seddon (1990), Brouwer (1992), Landgrebe (1994), Wake \& Baeder (1994) and references therein. An interesting review of these and other aspects is given by Conlisk (1997) who in particular notes the significant effects of wakes on individual blade flows in practice. Generally there continues to be a theoretical need to incorporate the multi-blade-wake nature of a real configuration, a need which is addressed by the present study and related ones on multiple-blade-wake interactions.

Theoretical works on multi-blade-wake interactions appear to be few, especially 
concerning boundary layers and allied features; see also Conlisk (1997). Smith \& Timoshin (1996a) investigated three-dimensional rotating configurations of thin blades (such as for a cut disk) under the assumptions of normal symmetry and negligible motion far from the configuration, and pointed out a number of unusual viscous-inviscid properties that arise despite the imposed uniform surface pressure distributions in their cases. The properties include double viscous layers and short/long interactions for various limits, for instance where each separate blade and its own viscous layer is embedded in a much thicker incident motion outside. Subsequently the planarflow study of Smith \& Timoshin (1996b) allowed for non-symmetry in the normal direction, this introducing a distinct inner-outer flow interaction at tiny angles of incidence for example, coupled with increased upstream influence. This is again with fixed surface pressure to the first approximation but the unknown pressure distribution at the next approximation couples fully with the unknown leading-order wake shape in controlling the dominant velocities in particular.

The above two papers further noted that a quasi-periodic local flow solution emerges, sufficiently downstream of the leading blade in the two-dimensional setting or for a sufficient number of blades on a three-dimensional rotor, for the limit of many (or multi) blades. In practice 'many' is found to mean 'more than about 4, thus indicating this case as being one of some practical value, and this is the case of most interest here. See also Hawkings \& Lowson (1974), Parry \& Crighton (1989), and Seddon (1990) concerning rotor acoustics and the actuator disk model used in industrial prediction. The same limit case of multi-blade motions was considered by Bowles \& Smith (2000) in the presence of interaction between the unknown blade or wake pressure and the unknown displacement of, or efflux into, the outer inviscid motion, but with normal symmetry and hence zero lift. This pressure-displacement interaction was incorporated to allow for the regular flow separations shown in their results, unlike in the previous Smith \& Timoshin $(1996 a, b)$ studies; and the interaction itself, combined with spatial periodicity, led to several distinct features including interactions covering an entire blade and a full coupling between shortblade effects and long-wake effects. The so-called condensed case was also covered, corresponding to fixed displacement with unknown pressure and covering a very wide range of length scales. The last paper in addition led to the study in Smith, Bowles \& Li (2000) of transition in near wakes, partly of the kind encountered by Bowles \& Smith.

The present concern is with the effects of non-symmetry in the normal direction on the viscous-inviscid interactive planar flow (with unknown pressure) past successive blades and wakes, and the implications for the lift and drag on the blade system. Again the multi-blade case is of most interest, with the comparatively thin boundary-layer and wake motion due to each separate blade being embedded well within an outer shear flow produced by the complete blade system. Indeed each blade is positioned relatively near the centreline of the preceding wake.

The distinction from the only previous study involving unknown-pressure interaction with multiple blades (Bowles \& Smith 2000) is partly obvious and partly not: obviously there is now the need to solve the flow equations both above and below the typical blade, generating unequal surface pressures and lift; not obvious, or less obvious, is the finding of jumps in the pressures, velocities and so on across the blade leading edge in the streamwise direction. That is, on the streamwise scale of the blade the flow solution is discontinuous at the leading edge. The reason for this pivotal assumption is clear from considering a single non-symmetric blade positioned within an oncoming smoothly profiled wake motion. This wake 
and the ensuing two boundary layers grown on the blade surfaces are thin layers, suggesting parabolic solution dependence locally and no upstream influence. A dilemma then arises at the trailing edge and in the subsequent wake, however, where normal continuity of pressure is required as in the Kutta condition and yet the two surface boundary layers in general would yield different trailing-edge pressures, due to parabolicity. The dilemma is resolved by the discontinuity in the (long-scale) solution at the leading edge, in the neighbourhood of which virtually all the necessary upstream influence is concentrated. A self-consistent discontinuous flow cannot be set up with the discontinuity fixed at any station other than the leading edge.

Further motivation and background for the above is provided by the experimental and computational findings presented in figure 8 of Conlisk (1997) for a lifting rotor in hover, which give indications of a notably abrupt pressure rise or fall near the leading edge on both surfaces of the blade, apparently more so than for usual isolated blades. Leading-edge pressure jumps in other configurations are also noted in the next paragraph. The discontinuity involved here is smoothed out within a shorter streamwise length scale surrounding the leading edge where substantial normal pressure gradients are generated, via an inviscid Euler flow region within which largely separation-free motion is assumed. The pressure jumps encountered in the current flows are supported by, on the one hand, the presence of forward streamwise uniform-shear flow, at relatively large normal distances above and below the leading edge, and on the other the solid surface itself. The unbounded shear flows in particular allow a pressure jump to be maintained through coupling with the jump in the mass flux (from the balancing of the inertial force proportional to streamwise shear against the streamwise pressure gradient), in contrast to unbounded uniform streams for example.

Alternative mechanisms for leading-edge jumps are described by Jones \& Smith (2000) and Smith \& Jones (2000) in the contexts of body-ground interactions and branching flows respectively. These likewise generate large streamwise and normal pressure gradients locally but with physical support provided by two or more containing walls instead. In the present configuration the pressure jumps found are similar to those experienced at a discontinuity of vorticity in vortex sheet dynamics, with continuous velocity, as described in Smith et al. (2000) recently.

The motion of concern here is taken to be two-dimensional, laminar and steady, for an incompressible fluid. Section 2 describes the flow scales, derived from an order-of-magnitude argument which emphasizes the range of the condensed-flow theory to be used, followed by the governing equations and boundary conditions. The latter include the main new feature of the pressure jump and its accompanying jump in mass flux, streamwise across the leading edge, the mechanism for which is discussed in $\S 3$. This is for the spatially periodic case associated with multiple blades, although it also extends to general configurations where a blade is aligned or nearly aligned with an oncoming wake containing comparatively low minimum velocities, as with rotor-stator row interactions in turbomachinery for instance. Section 4 presents computational results for the present multi-blade flows with various degrees of nonsymmetry, followed by an analysis for small ratios of blade length to wake length in $\S 5$ which is motivated by the short-blade approach developed in Bowles \& Smith (2000) and obtains explicit results for the surface pressures, the wake shape and the lift generated, for comparison. The theoretical results are remarkably similar to the experimental and numerical ones in Conlisk (1997, figure 8) mentioned previously. Section 6 provides further comments. 


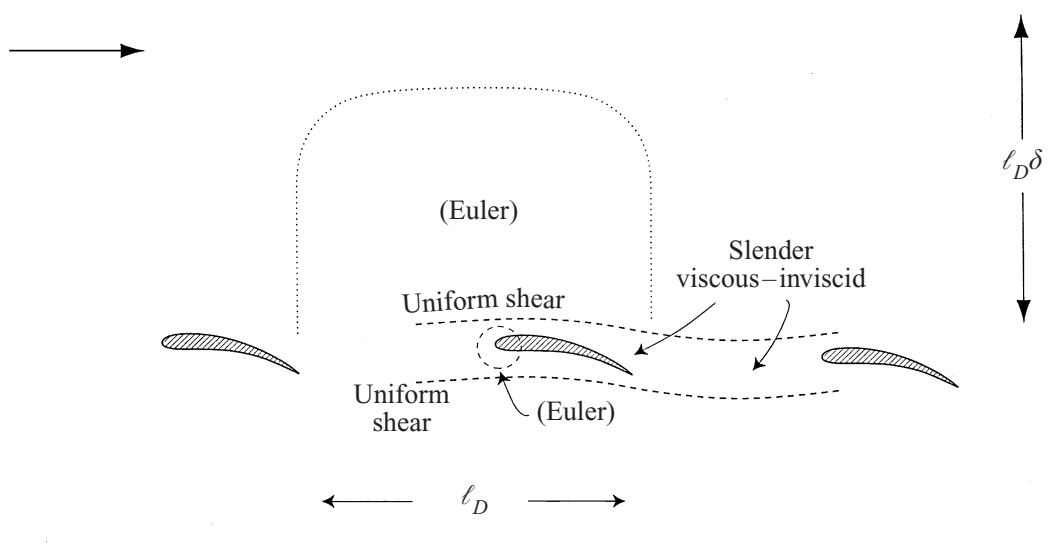

FIGURE 1. Diagram of the multi-blade and wake flow, with normal non-symmetry, indicating the slender viscous-inviscid layers and accompanying non-slender Euler regions near the leading edges as well as on a broader scale.

\section{The flow structure and main governing equations}

2.1. Scales and range of the theory

We start with an order-of-magnitude reasoning. The thin non-symmetric blade and its wake, representative of the multiple blade configuration, are taken to be of comparable characteristic length $\ell_{D}$, aligned or nearly aligned with the uniform free stream of speed $u_{D}$ and buried within the surrounding incident total boundary layer or wake of thickness $\ell_{D} \delta$ in the normal direction. The subscript $D$ denotes a dimensional quality. The ratio $\delta$ may be small, large or of order unity but it is assumed to be much greater than the classical thickness ratio of order $R e^{-1 / 2}$ associated with a single isolated thin blade in a uniform stream, where $R e=u_{D} \ell_{D} / v_{D}$ is the Reynolds number and $v_{D}$ is the kinematic viscosity. This assumption corresponds to there being a large number of blades present, either in the pure two-dimensional setting with the leading blade lying far upstream or in the rotating-flow arrangement (Smith \& Timoshin; Bowles $\&$ Smith), this leading to the build-up of thickness of the surrounding viscous motion as it passes over the many other blades which altogether cover a streamwise extent much larger than $\ell_{D}$.

Likewise the blade thickness is taken to be much less than the surrounding incident flow thickness $\ell_{D} \delta$. In consequence the surrounding streamwise velocity profile near the blade is linear, giving velocities of order $u_{D} \delta_{1} / \delta$ in the typical viscous sublayer of thickness $\ell_{D} \delta_{1}$ on the blade and in its wake, and hence the inertial force there is of order $\rho_{D} u_{D}^{2} \delta_{1}^{2} /\left(\delta^{2} \ell_{D}\right)$ whereas the viscous force has characteristic size $\mu_{D}\left(u_{D} \delta_{1} / \delta\right) /\left(\ell_{D} \delta_{1}\right)^{2}$, with $\rho_{D}, \mu_{D}$ being the constant fluid density and viscosity respectively. A balance of these two forces determines the viscous thickness ratio $\delta_{1}$ as $\left(\operatorname{Re}^{-1} \delta\right)^{1 / 3}$. The corresponding velocity, of order $u_{D} \delta_{1} / \delta$, then points to a viscous pressure response $p_{D V}$ of order $\rho_{D} u_{D}^{2} \delta_{1}^{2} / \delta^{2}$, i.e. $p_{D V} \sim \rho_{D} u_{D}^{2} R e^{-2 / 3} \delta^{-4 / 3}$, on the blade. This estimate presupposes the local sublayer flow response to be nonlinear and viscous to accommodate the mixed blade-wake conditions and the blade thickness to be of size $O\left(\ell_{D} \delta_{1}\right)$ at most. 
Outside the viscous sublayer, on the other hand, the normal displacement effect of representative size $\ell_{D} \delta_{1}$ needs consideration, over a normal distance of extent $\ell_{D}$, where it induces an inviscid pressure response $p_{D I}$ say. If the ratio $\delta$ is small then potential-flow properties in the free stream establish that $p_{D I} \sim \rho_{D} u_{D}^{2} \delta_{1}$, due to the displacement slope, and the same estimate holds when $\delta$ is of order unity, while if $\delta$ is large $p_{D I} \sim \rho_{D} u_{D}^{2} \delta_{1} / \delta^{2}$ because of the surrounding shear. So for the former estimate the inviscid pressure would be much larger than the viscous pressure if $\delta_{1} \gg R e^{-2 / 3} \delta^{-4 / 3}$, i.e. if $\delta \gg R e^{-1 / 5}$. In such a case the argument is that the displacement slope must remain negligible in order to maintain the two pressures at the same level, given that $p_{D V}$ is independent of normal distance provided the viscous sublayer is slender, its slope being $\delta_{1}$ which is small provided $\delta \ll R e$. Moreover the comparison of the pressures above when $\delta$ is instead large also requires $\delta_{1}$ to be small. See figure 1 which provides a sketch of the flow configuration, the main scales and the setting.

Accordingly the theory applies for the entire wide range $R e \gg \delta \gg R e^{-1 / 5}$ of surrounding thicknesses $\delta$, and it demands that the slope of the local viscous displacement remains negligible. The requirements

$$
R e \gg \delta \quad \text { and } \quad R e \gg \delta^{-5}
$$

alternatively express the range of validity in terms of Reynolds numbers, for a given ratio $\delta$ of the surrounding flow thickness to the representative blade-wake length. (Interpreting this practically, if $\delta$ is about $\frac{1}{5}$ for instance then Re must be greater than about $1.25 \times 10^{4}$, whereas if $\delta$ is about 5 then $R e$ must exceed only about 20 ; here ' $\gg$ ' is interpreted as 'is at least four times larger than'.)

2.2. Governing equations for the multiple periodic boundary layers and wakes

In consequence of $\S 2.1$ the governing equations of the thin viscous boundary layer and wake of the typical blade are

$$
\begin{gathered}
\frac{\partial u}{\partial x}+\frac{\partial v}{\partial y}=0 \\
u \frac{\partial u}{\partial x}+v \frac{\partial u}{\partial y}=-p^{\prime}(x)+\frac{\partial^{2} u}{\partial y^{2}} .
\end{gathered}
$$

These are subject to the conditions of no slip on the upper and lower blade surfaces, regularity in the wake(s), zero displacement slope, and streamwise periodicity, in the form

$$
\begin{gathered}
u=v=0 \quad \text { at } \quad y=f^{ \pm}(x), \quad 0<x<\ell, \\
u, v, p \text { regular in wakes, } \\
u \sim \pm \lambda^{ \pm} y+c^{ \pm} \text {as } \quad y \rightarrow \pm \infty, \\
L \text {-periodicity in } x,
\end{gathered}
$$

respectively. Here the superscripts \pm refer to the areas above and below the blade (or the wake centreline), in turn. Further, the velocity is $u_{D} \delta_{1} \delta^{-1}\left(u, v \delta_{1}\right)$ in Cartesian coordinates $\ell_{D}\left(x, \delta_{1} y\right)$ (streamwise, normal in turn), the pressure is $\rho_{D} u_{D}^{2} \delta_{1}^{2} \delta^{-2} p$, and the blade and wake lengths are respectively $\ell_{D} \ell, \ell_{D}(L-\ell)$ with $\ell, L$ of order one, where $\delta_{1} \equiv\left(\operatorname{Re}^{-1} \delta\right)^{1 / 3}$ from above. The origin is placed at the blade leading edge for convenience. The non-symmetric blade surfaces are specified by $f^{ \pm}$in $(2.3 a)$, where $f^{+} \geqslant f^{-}$(and $f^{ \pm}(0)=0$ ), the blade thickness scaling here on $\ell_{D} \delta_{1}$. In $(2.3 c)$ the 
order-one positive constants $\lambda^{ \pm}$represent the given incident shears, and the unknown order-one constants $c^{ \pm}$reflect the effective absence of displacement slope required in the theory for the range (2.1); their being non-zero and unequal in general is explained by the surrounding longer-scale evolution as in Bowles \& Smith, involving for each of the lower and upper half-planes an outer thicker viscous layer wherein the displacement effects are smoothly reduced to zero at sufficiently large normal distances. The constraint $(2.3 d)$ of local spatial periodicity is necessary to preserve the overall flow structure with its underlying dependence on two streamwise length scales, the local one $\ell_{D}$ and the larger more global one associated with the surrounding motion as described earlier.

\subsection{The discontinuity}

A further important (and unusual) feature is also relevant, however, as a streamwise pressure jump must be allowed at the leading edge. This is due to the local parabolicity of the boundary layers and wakes in (2.2) and $(2.3 a-c)$, indicating zero upstream influence in general and therefore a possible contradiction with the equi-pressure requirement within $(2.3 b)$ at the trailing edge: the boundary layers on the upper and lower surfaces are different from each other and so they usually produce unequal trailing-edge pressures if they begin with identical leading-edge pressure. The resolution is provided by a flow solution discontinuity, which can occur self-consistently only in the neighbourhood of the leading edge (see next section), where all the upstream influence is focused, in a sense. So in general the scaled pressure $p(0-)$ just ahead of the leading edge is different from the two distinct values $p^{ \pm}(0+)$ on the upper and lower surfaces just downstream. Instead the Bernoulli quantity $p+\frac{1}{2} u^{2}$ and the vorticity are conserved across the leading edge, yielding the condition(s)

$$
\begin{gathered}
\left(p+\frac{1}{2} u^{2}\right)(\text { at } x=0-)=\pi^{+}+\frac{1}{2}\left(u^{+}\right)^{2}=\pi^{-}+\frac{1}{2}\left(u^{-}\right)^{2}, \\
\frac{\partial u}{\partial y} \text { is conserved along streamlines. }
\end{gathered}
$$

Here $\pi^{+}$denotes the unknown value of $p^{+}$at $x=0+, u^{+}$is the unknown incident slip velocity felt at the leading edge $(x=0+$ again) by the blade flow on the upper surface, provoking a Blasius-like sublayer there, and similarly for $\pi^{-}, u^{-}$.

The quantity $u$ of the upstream wake at $x=0-$ in $(2.3 e)$ is to be evaluated at a $y$-position, to be found, such that for continuity the value of $\psi$ at that $y$-position is equal to the $\psi$ value on the current blade in $x>0$; the stream function $\psi$ here satisfies $u=\partial \psi / \partial y, v=-\partial \psi / \partial x$ as usual. All the incoming upstream-wake streamlines are therefore displaced, on passing through the jump, so as to satisfy the vorticity conservation requirement $(2.3 f)$ on the (outgoing) streamlines above and below the blade. The jump features of $(2.3 e, f)$ are discussed in more detail in the next section.

\section{The leading-edge jumps}

\subsection{Local Euler region}

The streamwise length scale involved in the comparatively short region necessary to smooth out the leading-edge jumps of $(2.3 e, f)$ is of order $\ell_{D} \delta_{1}$, the same as the normal sublayer scale, and the sizes of the velocity (both components) and pressure are clearly $u_{D} \delta_{1} / \delta, \rho_{D}\left(u_{D} \delta_{1} / \delta\right)^{2}$ respectively. So the leading-edge region is controlled 
by the Euler equations in $(\boldsymbol{u}, p)(\bar{x}, y)$, for $|y|<\infty$ and $|\bar{x}|<\infty$ where $x=\ell_{D} \delta_{1} \bar{x}$, with the thin blade appearing as a flat plate at a constant $y$ value for all positive $\bar{x}$ say.

Hence the vorticity and Bernoulli quantities are conserved along the flow streamlines, yielding

$$
\nabla^{2} \psi=g_{1}(\psi), \quad p+\frac{1}{2}|\boldsymbol{u}|^{2}=g_{2}(\psi)
$$

where $\nabla^{2}$ denotes $\partial_{\bar{x}}^{2}+\partial_{y}^{2}$ and the functions $g_{1}$ (negative vorticity), $g_{2}$ (pressure head) are fixed as usual by the wake velocity profile and pressure level entering upstream and are related by $g_{1}=\mathrm{d} g_{2} / \mathrm{d} \psi$. The flow is supposed to enter and leave the present region uni-directionally but with an overall displacement of its streamlines and vorticity distribution accompanied by pressure changes, consistent with the upstreamto-downstream jumps in $(2.3 e, f)$, and with smooth attached flow in between such that $\psi$ is an unknown constant on the quasi-semi-infinite blade. A thin viscous boundary layer is generated of course on the local leading-edge surface here and this is supposed to remain broadly attached, for some surface shapes at least, before forming downstream the beginnings of the Blasius-like effects on the longer scale described just after (2.3f). Large-scale leading-edge separations are thus discounted.

The upstream and downstream asymptotes for (3.1) at large $|\bar{x}|$, where the scaled normal velocity $-\partial \psi / \partial \bar{x}$ tends to zero and $\nabla^{2} \psi$ tends to $\partial u / \partial y\left(=g_{1}\right)$, yield the conditions $(2.3 e, f)$ exactly, then, although the solution of (3.1) for all $\bar{x}, y$ is a numerical task generally for an arbitrary distribution $g_{1}(\psi)$.

The far-field solution of (3.1) can be found however, since $g_{1}$ then tends to $\pm \lambda^{ \pm}$ and so

$$
\begin{gathered}
\psi \sim \pm \frac{1}{2} \lambda^{ \pm} y^{2}+c^{ \pm} y+\left[a_{3}+b_{3} \theta\right], \\
p \sim \pm \lambda^{ \pm}\left[a_{3}+b_{3} \theta-\frac{1}{2} b_{3} \sin 2 \theta\right]+c_{3}^{ \pm} .
\end{gathered}
$$

Here $\theta$ is the polar angle $\tan ^{-1}(y / \bar{x})$ and, for positive $y, a_{3}=q_{2}, b_{3}=\left(q_{1}-q_{2}\right) / \pi$, while, for negative $y, a_{3}=2 q_{1}-q_{2}, b_{3}=\left(q_{2}-q_{1}\right) / \pi$, with $q_{1}, q_{2}$ denoting respectively the constant values of $\psi$ far upstream at fixed $y$ and on the blade. This is consistent with $(2.3 c)$. The pressure results (3.2b) follow from $g_{2} \sim \pm \lambda^{ \pm} \psi$ along with (3.1b), and the constants $c_{3}^{ \pm}$are to ensure pressure continuity upstream. The pressures involved in the jumps of $\S 2$ (from $\bar{x}=-\infty$ to $+\infty$ ) are therefore related by

$$
\pi^{ \pm}-p(0-)= \pm \lambda^{ \pm}\left(q_{2}-q_{1}\right)
$$

from the values of $b_{3}^{ \pm}$above, consistent with $(2.3 c, e, f)$. The far-field behaviour above is smooth for $\theta$ between zero, $\pi$ and between $\pi, 2 \pi$, and this is completed by layers with width $y$ of order unity upstream and downstream at large positive and negative $\bar{x}$. These two layers merge in turn with the downstream boundary layer and the upstream wake of $\S 2$.

\subsection{An exact local solution}

Further analytical guidance is provided by the following case, allowing for nonsymmetry. Suppose the upstream wake profile has $u$ given by $u_{0}+\lambda^{+} y$ for all positive $y$ and $u_{0}-\lambda^{-} y$ for all negative $y$ where $u_{0}$ is a positive constant, so that $u$ is continuous and positive, with pressure level $p=p_{0}$ say, and $\psi$ again tends to $q_{1}$ at $y=0$ upstream as $\bar{x} \rightarrow-\infty$ but $\psi$ is equal to $q_{2}$ on the blade which occupies $y=0$ for all positive $\bar{x}$. Here we are taking $c^{ \pm}$to be zero. The motion has to be non-symmetric in $y$ if $q_{1} \neq q_{2}$. The vorticity then gives $\nabla^{2} \psi$ equal to $\pm \lambda^{ \pm}$above and below the streamline $\psi=q_{1}$ respectively, while at that streamline for all negative $\bar{x}$ continuity of $\psi, p$ is 


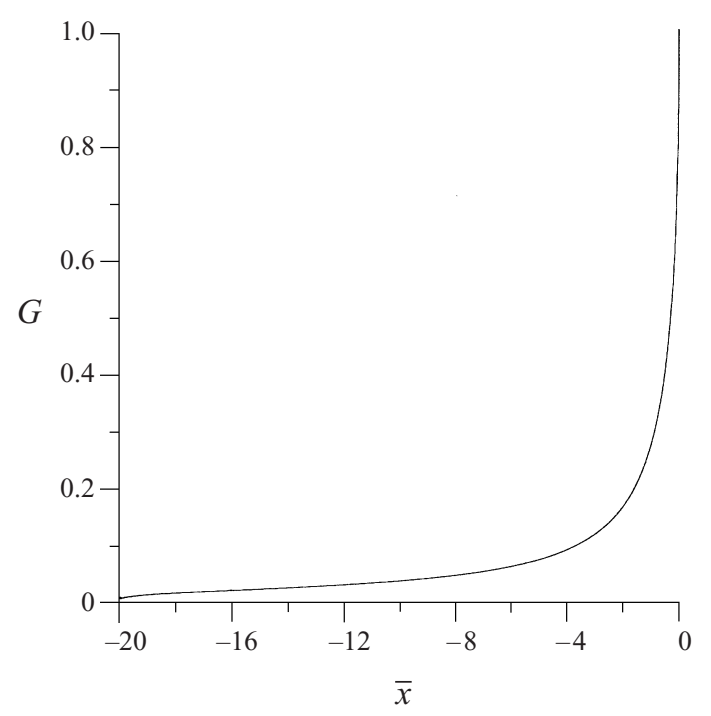

Figure 2. Solution of (3.4) for $G$ vs. negative $\bar{x}$, with $\gamma=\bar{q}=1$. The behaviours at small $|\bar{x}|$ and large $|\bar{x}|$ are $1-O\left(|\bar{x}|^{1 / 2}\right)$ and $O\left(|\bar{x}|^{-1}\right)$ respectively.

required. Hence $\bar{\psi} \equiv\left(\psi \mp \frac{1}{2} \lambda^{ \pm} y^{2}-u_{0} y-q_{1}\right)$ must satisfy Laplace's equation and is expected to be bounded in the far field. If in addition $q_{2}-q_{1}$ is small then the other boundary conditions are that $\bar{\psi}=\bar{q}\left(\equiv q_{2}-q_{1}\right)$ on $y=0 \pm$ in $\bar{x}>0$ and, on $y=0 \pm$ in $\bar{x}<0, \bar{\psi}$ and $\left( \pm \lambda^{ \pm} \bar{\psi}-u_{0} \partial \bar{\psi} / \partial y\right)$ are continuous. These two continuity conditions can be replaced by $\gamma \bar{\psi}=\partial \bar{\psi} / \partial y$ at $y=0, \bar{x}<0$ as far as solving for $\bar{\psi}$ in the upper half-plane is concerned, with symmetry $\bar{\psi}(\bar{x},-y)=\bar{\psi}(\bar{x}, y)$ for all positive $y$ but noting that $\partial \bar{\psi} / \partial y$ is not identically zero at $y=0 \pm$. Also the constant $\gamma \equiv\left(\lambda^{+}+\lambda^{-}\right) /\left(2 u_{0}\right)$ is positive, and the far-field behaviour has $\bar{\psi} \sim \bar{q}(\pi-\theta) / \pi$, in keeping with the jump in $\psi$ from upstream to downstream. Hence $\tilde{\psi} \equiv \bar{\psi}+\bar{q}(\theta-\pi) / \pi$ must also satisfy Laplace's equation in the upper half-plane but $\tilde{\psi}$ is to be zero at $y=0$ for positive $\bar{x}$ and to satisfy $\gamma \tilde{\psi}=\partial \tilde{\psi} / \partial y-\bar{q} /(\pi \bar{x})$ at $y=0$ for negative $\bar{x}$. So the function $G(\bar{x})$ defined by $\tilde{\psi}$ evaluated at $y=0$ is identically zero for $\bar{x}>0$ but unknown for $\bar{x}<0$ and, as it yields $\partial \tilde{\psi} / \partial y$ at $y=0$ in terms of a Cauchy-Hilbert integral of $G^{\prime}(\bar{x})$, the function is governed by the integro-differential equation

$$
\gamma G(\bar{x})+\frac{1}{\pi} \mathrm{PV} \int_{-\infty}^{0} \frac{G^{\prime}(\xi) \mathrm{d} \xi}{\bar{x}-\xi}=0,
$$

for all $\bar{x}<0$, subject to

$$
G(0-)=\bar{q}, \quad G(-\infty)=0 .
$$

The constraint $(3.4 b)$ is to keep the original stream function continuous at the leading edge in the present scales and $(3.4 \mathrm{c})$ stems from the far-field response. The implied jump in $G(\bar{x})$ across $\bar{x}=0 \pm$ means that the term $-\bar{q} /(\pi \bar{x})$ is absorbed into the principal-value (PV) integral of (3.4a).

The solution of (3.4) was determined computationally by a finite difference procedure and is shown in figure 2, normalized such that $\gamma, \bar{q}$ are unity. This numerical solution agrees closely with the analytical one of Stewartson (1960) (kindly pointed out to us by Professor Susan Brown), to well within the three significant figures that he quotes. We observe that the decay $G \sim-\bar{q} /(\pi \gamma \bar{x})$ at large negative $\bar{x}$ is slow. 
The exact solution of (3.4) relies on $\gamma$ being positive and finite, as well as on $\bar{q}$. Thus the incident shears $\lambda^{ \pm}$are again seen to be central to the flow structure, as is the non-zero minimum wake velocity $u_{0}$ in this special case. We see moreover that $\psi, p$ jump from $\left( \pm \frac{1}{2} \lambda^{ \pm} y^{2}+u_{0} y+q_{1}\right), p_{0}$ respectively upstream at large negative $\bar{x}$ to $\left( \pm \frac{1}{2} \lambda^{ \pm} y^{2}+u_{0} y+q_{2}\right), p_{0} \pm \lambda^{ \pm}\left(q_{2}-q_{1}\right)$ respectively downstream at large positive $\bar{x}$, at a fixed positive or negative $y$ value. These overall jumps are in line with $(2.3 e, f)$ and (3.3), and likewise the smooth local flow solution near the leading edge associated with $(3.4 a)$ is exactly in line with (3.1) and (3.2).

\subsection{Larger Euler region}

There is also a larger outer Euler-like zone but of linearized flow, of course, anticipated near the start of $\S 2$. Its response to the $\psi, p$ variations of $(2.2)$ and (2.3) is worth considering and interpreting, especially given the jumps of $(2.3 e, f)$ and (3.3). The outer zone has streamwise and normal dimensions both of order $\ell_{D}$ and so its perturbation stream function or velocity is controlled by Laplace's equation if the ratio $\delta$ is smaller or larger than $O(1)$ and by Rayleigh's equation with zero wave speed if $\delta$ is of $O(1)$. The boundary conditions require $L$-periodicity in $x$, boundedness at relatively large normal distances and matching with $\psi, p(x)$ of (2.2) and (2.3) at small normal distances, supplemented by the match with (3.2) at $O(1)$ values of $\theta$ near the origin. The flow solution can be written down explicitly when $\delta$ is small or large and it confirms that the required source- or sink-like jump in $\psi$ across $x=0 \pm$, linked with the pressure jump of (3.3) by a factor $\pm \lambda^{ \pm}$, is met by an overall perturbation mass flux vertically through the multi-blade system, above the centreline, and a similar flux below. These two fluxes are different in general because of the intervening streamwise convection (of (2.2)) in the viscous flow near the blades and their wakes. A corresponding interpretation holds also when the ratio $\delta$ is of order unity.

\section{Computational solutions}

\subsection{Numerical method}

The flow field for (2.2) and (2.3) divides computationally into the three areas $f^{+} \leqslant$ $y \leqslant y_{1}$ above the blade for $0<x<\ell, f^{-} \geqslant y \geqslant y_{2}$ below the blade for $0<$ $x<\ell$, and $y_{3} \leqslant y \leqslant y_{4}$ in the wake for $\ell<x<L$. Here $y_{1}, y_{4}\left(y_{2}, y_{3}\right)$ are suitably large and positive (negative). The flow in each area was resolved numerically with a finite-difference procedure as in Bowles \& Smith (2000), using a Prandtl shift with $y-f^{ \pm}$replacing $y$ for convenience in the first two areas; the junctions between the areas are considered in the next two paragraphs. In the procedure three-point centred differencing is applied throughout in $y$ with step length $\Delta y$ and two-point in $x$ for $(2.2)$. This yields, with $(2.3 a, c)$ or $(2.3 b, c)$ and with set $c^{ \pm}$values, a pentadiagonal system from which the velocities and pressure $\left(p^{+}, p^{-}\right.$or $\left.p\right)$ at a discrete station $x$ are found semi-implicitly for all the given discrete $y$ values, from the solution at the previous station $x-\Delta x$, where $\Delta x$ is the streamwise step length. Forward flow with $u$ positive is assumed for now. Second-order accuracy in $x$ as well as $y$ is then obtained by the double-stepping scheme of Smith \& Timoshin $(1996 a, b)$. The solution behaviours immediately after the leading and trailing edges are also accommodated by their approach and that of Bowles \& Smith, as, with minimal linkage to other $x$ positions through the streamwise derivatives, the viscous terms at the current $x$ position act to suppress the grid-like oscillations that can 
occur in other rectangular-grid approaches proceeding from a leading or trailing edge. The local flow solutions merge very closely over a streamwise step or two to the known forms of Blasius and Goldstein essentially, in surface shear, pressure and so on.

The solution is marched forward through the two areas above and below the blade, then on through the wake area and on around the next blade (see below), and so on repetitively until spatial $L$-periodicity of the velocities and the pressure gradient is ensured to within a given tolerance, which is typically $10^{-3}$ and is attained within about $7-10$ blade sweeps. The pressure itself is also required to be $L$-periodic however, subject to the jump conditions across the leading edge that are about to be described, and this requirement sets one constraint on the two unknown constants $c^{ \pm}$. The requirement is met by an application of Carter's method similar to that in Bowles \& Smith, leading to a total of approximately 25 iterative sweeps.

During each sweep the jump in pressure $p$ and its accompanying profile changes in $\psi, u$ at the blade leading edge are handled as follows. The vorticity requirement $(2.3 f)$ amounts to there being first a uniform shift $y_{s}$ in all the $y$ values with the wake $u$ profile otherwise unchanged, as the leading edge is passed over, and second an adjustment in the $u$ profile which must be made above the blade and similarly an adjustment, usually different, below the blade, in order to cater for the two outer constraints in $(2.3 c)$. The constants $c^{ \pm}$are already set at this stage, we recall. These alterations in the $u$ profiles consistent with $(2.3 f)$ serve to fix the two pressures $p^{ \pm}=\pi^{ \pm}$at $x=0+$ in view of $(2.3 e)$.

When the shear is nearly uniform or the $y_{s}$ value is relatively large, or both, for instance, the outer constraints (2.3c) imply that, as the jump $y \rightarrow y+y_{s}$ holds across the leading edge in the downstream direction, $u \rightarrow u \pm \lambda^{ \pm} y_{s}$ and so then (2.3e) yields the explicit results

$$
\pi^{ \pm}=p(0-) \mp \lambda^{ \pm} y_{s} u_{0}\left(-y_{s}\right)-\frac{1}{2}\left(\lambda^{ \pm}\right)^{2} y_{s}^{2}
$$

for the upper and lower surface pressures immediately after the leading edge. This approximation was used to guide the present numerical reasoning, as well as proving helpful in the next section. In practice normal compression or expansion of the velocity profile is incorporated in the numerical method by means of interpolation, in order to satisfy $(2.3 f)$. Hence next, given $y_{s}$, the flow solutions for the velocities and pressures in the areas above and below the blade can be marched forward to the trailing edge. There, at $x=\ell$, the pressures $p^{ \pm}(\ell)$ for the start of the wake area must be equal however because of $(2.3 b)$ and so an iteration is performed, adjusting the scaled angle of attack $\alpha$ (which is built into the shapes $f^{ \pm}$) to produce equal $p^{ \pm}(\ell)$ values from the leading- to trailing-edge marches. The alternative of specifying $\alpha$ and deducing the shift $y_{s}$ is rather more cumbersome. The wake march then proceeds from $x=\ell(+\Delta x)$ to $x=L$, beginning with the velocity profiles supplied from above and below the blade and ending at the next leading edge, ready for the next $y_{s}$ shift there.

The entire process above is sufficient to leave only one constant undetermined, say $c^{+}$, which is an input parameter related to the vertical through-flow of the overall system: see $\S 3$. The alternative of setting $c^{ \pm}$equal was also explored. The positive shear constants $\lambda^{ \pm}$were taken as unity in all our computations, and the grids were uniform. Reversed flows with $u$ negative were treated by windward differencing in the streamwise direction. Typical grids used had $(\Delta x, \Delta y)$ equal to $(0.005,0.025)$, $\left(y_{1}, y_{2}, y_{3}, y_{4}\right)$ equal to $(20,-20,-20,20)$, and finer grids were adopted as checks. 

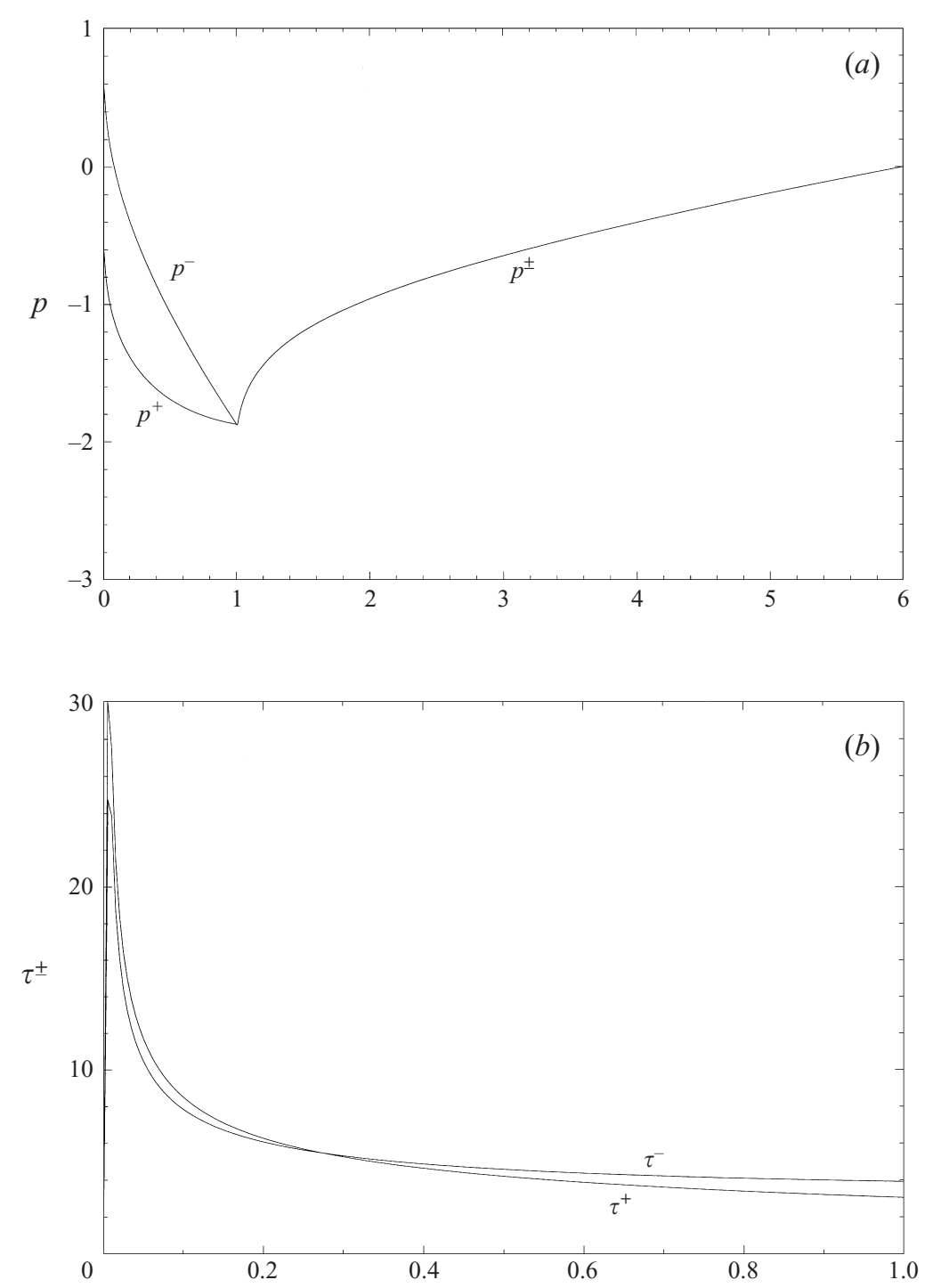

FIGURE 3. Computational flow solution for flat-plate blade case of $f^{ \pm} \equiv-\alpha x$ at scaled incidence angle $\alpha=0.258$. (a) Blade pressures $p^{ \pm}$and wake pressure $p$ vs. $x(b)$ scaled skin frictions vs. $x$, on the blade. Here, and in figures $4-10, \ell$ is normalised to be one and the ratio $r(=\ell / L)$ is $1 / 6$.

\subsection{Results}

The results are presented in figures 3-10 for a ratio $r$ of the blade length to the spatial period equal to $1 / 6$, i.e. $r \equiv \ell / L$ is $1 / 6$ here. Further, dividing $x, y, p, u, \tau$ by $\ell^{m}$ with $m$ equal to $1,1 / 3,2 / 3,1 / 3,0$ respectively, and multiplying $c^{+}, c^{-}, f$ and scaled angles by $\ell^{n}$ with $n$ equal to $1 / 3,1 / 3,1 / 3,2 / 3$ in turn, shows that the factor $\ell$ may be normalized to 1 . So the results are given without loss of generality for blade lengths $\ell$ of unity and the spatial period $L$ equal to 6 , with a variety of other conditions. For clear record, the values of $\left(c^{+}, c^{-}, y_{s}\right)$ in figures $3-9$ are $(2,2,0.2),(2,2,0.25),(4.5,2.5,0.25),(4.55,2.55,0.125),(3.55,1.55,0.125)$, $(3.325,1.325,0.25),(0,0.1,0.25)$, respectively, and the corresponding values found 
(a)

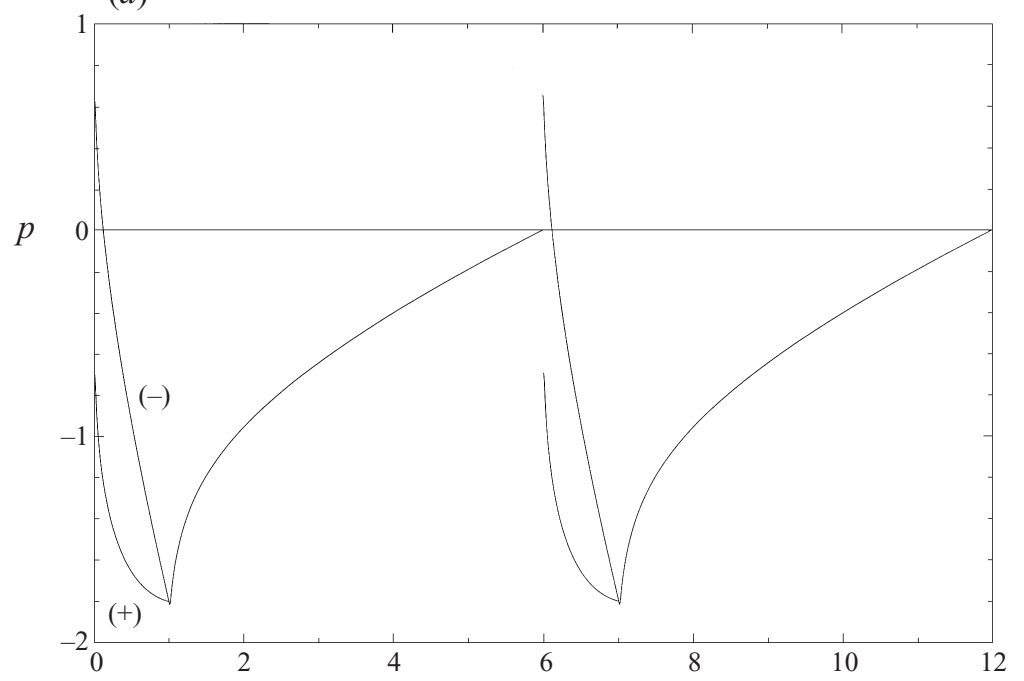

(b)

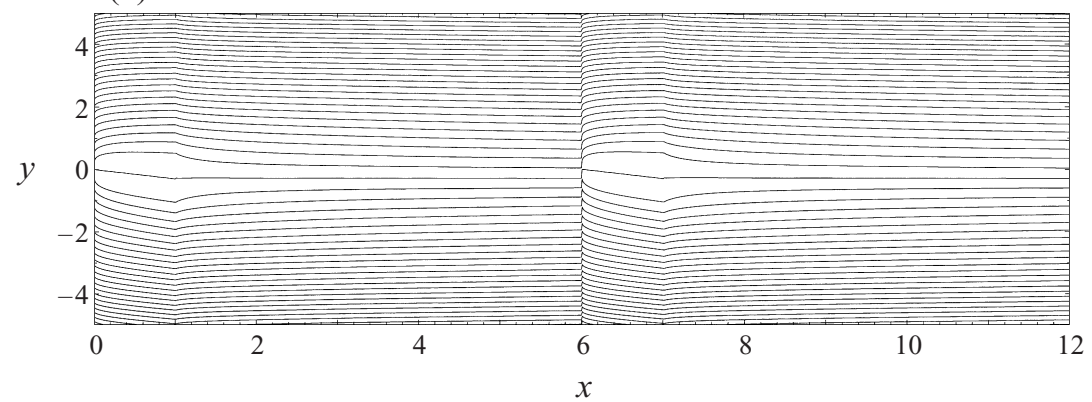

Figure 4. (a) Pressures and (b) flow streamlines over two spatial periods. Here $f^{ \pm} \equiv-\alpha x$, $\alpha=0.292$.

for $\left(\pi^{+}, \pi^{-}\right)$are $(-0.60,0.57),(-0.69,0.65),(-0.77,0.73),(-0.34,0.33),(-0.24,0.23)$, $(-0.42,0.38),(-0.34,0.29)$, to two decimal places.

Figure $3(a, b)$ is for the case of the flat-plate blade at scaled incidence $\alpha$, with equal constants $c^{ \pm}$imposed as 2.0 (and $y_{s}$ set at 0.2 ), while the calculated pressure constants $\pi^{ \pm}$at the leading edge are $-0.60,0.57$ respectively. The pressures on the blade and in the wake and the scaled skin frictions $\tau^{ \pm} \equiv \pm \partial u / \partial y$ at $y=f^{ \pm}$on the blade are shown. The blade flows are attached with forward motion. The pressure gradient is more favourable overall on the lower surface than on the upper, this being linked with the leading-edge pressure jumps, and so the positive $\tau^{-}$surface shear values slightly exceed the positive $\tau^{+}$values over the last $70 \%$ of the blade chord. The pressure difference also produces lift. The wake effect from the blade continues downstream to influence the next blade flow. Figure 4 $(a, b)$ likewise has flat plates at incidence, with the shift value $y_{s}$ at 0.25 , but pressures and streamlines are presented over two spatial periods in order to bring out the leading-edge jumps more explicitly. The effects found are broadly similar to those for the case of figure 3 . The pressures and streamlines in figure $5(a, b)$ are for almost the same incidence and $y_{s}$ is again set at 0.25 but now $c^{ \pm}$are unequal and the results further show the streamlines from 
(a)

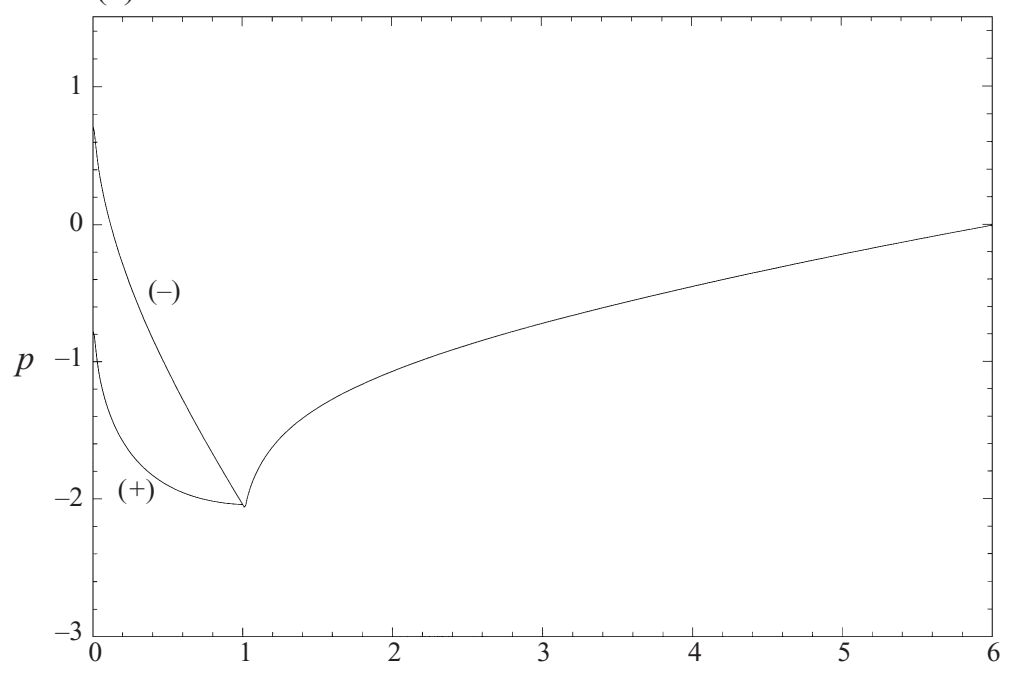

(b)

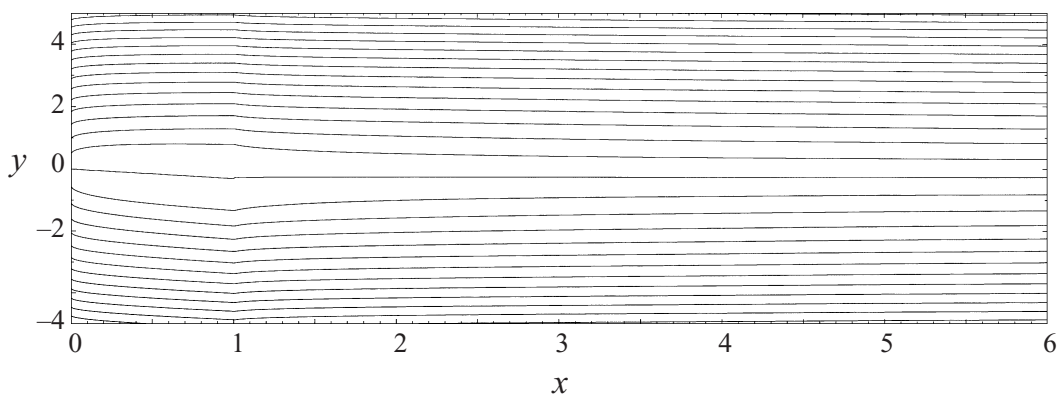

Figure 5. ( $a$ ) Pressures and (b) streamlines for the case $f^{ \pm} \equiv-\alpha x$, at incidence $\alpha=0.299$; the streamline from the blade $(0,1)$ misses the next blade $(6,7)$.

one blade ( $x$ between 0 and 1) clearly missing the leading edge of the next blade ( $x$ between 6 and 7). The influence on blade pressures as well as scaled surface shears and local streamline displacements is more pronounced than before.

The effects of blade thickness are included in the next four figures. For the smallest thickness, figure $6(a, b)$ has a $y_{s}$ value of 0.125 and it yields $\pi^{ \pm}$equal to $-0.34,0.33$ in turn for constants $c^{ \pm}$of $4.55,2.55$ respectively. Here the pressure gradient on each blade surface becomes adverse over the last $40 \%$ or so of the chord, the overall pressure variation is enhanced and the streamline displacement is increased. Close-up views of the streamlines as well as pressures are plotted in figure $7(a, b)$ for a thicker shape. The pressure variation on the blade is enhanced even more now, leading to stronger adverse pressure gradients near the trailing edge and more displacement of the local streamlines. Figure $8(a, b)$ presents the pressures and streamlines for a still thicker case which gives $\pi^{ \pm}$as $-0.42,0.38$ for $c^{ \pm}$equal to $3.325,1.325$ respectively. The trends in the scaled pressure and streamline displacement are accentuated from those in the preceding figure. These last two cases, unlike the previous ones, induce separated (reversed) flows as shown on at least one surface of each blade. See Smith (1983) and Jones \& Smith (2000). The thickest shape is in figure $9(a-c)$, for which $\pi^{ \pm}$ 


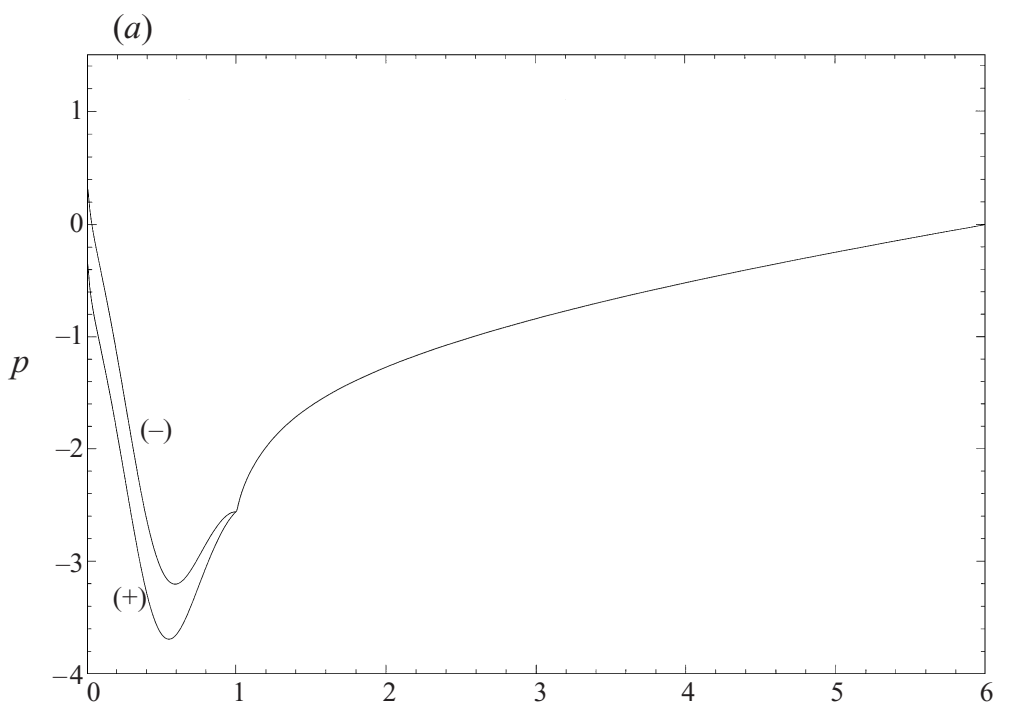

(b)

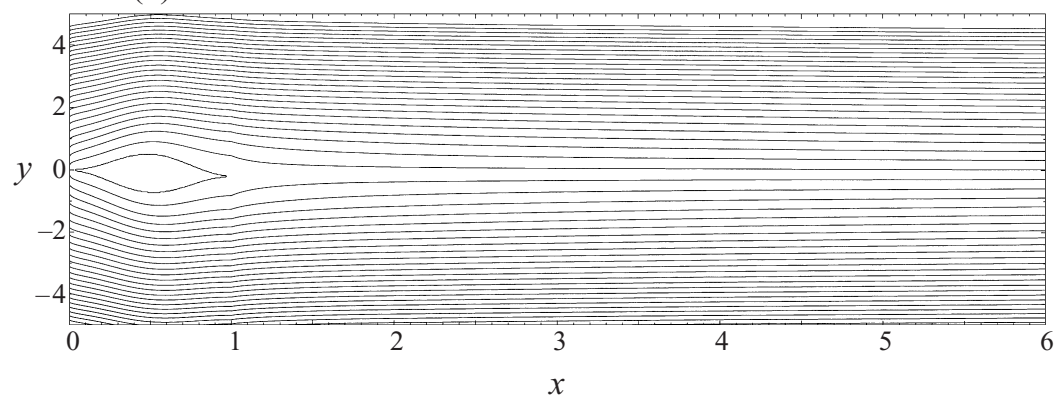

Figure 6. (a) Pressures and (b) streamlines for blade shape $f^{ \pm}= \pm 2 f^{*} \sin ^{2}(\pi x / \ell)-\alpha x$ with $f^{*}=0.3$ and scaled incidence $\alpha=0.210$.

are $-0.34,0.29, c^{ \pm}$are $0,0.1$ and the values of $p^{ \pm}$found at the trailing edge are both just less than -2.60 . This provokes the largest separation eddy(ies), as expected. On each blade surface the scaled surface shear displays a positive minimum followed by a positive maximum and then becomes negative as separation occurs before the trailing edge, in line with the increased adverse pressure gradients. The local displacement of the streamlines is also increased. In all cases the pressure gradient in the wake is adverse but relatively mild.

The jumps across the leading edge are apparent throughout, along with the boundary layer growth on each blade surface and the thin wake downstream. The above solutions for pressure in particular compare favourably in qualitative terms with the experimental and computational results in figure 8 of Conlisk (1997) for a lifting rotor in hover mode. The lift and drag for the flat-plate cases of figures 3-5 are given in figure 10 in scaled form, from integration of the surface pressure differences and the skin friction sums along the blade. The scaled lift increases monotonically with the scaled angle of incidence $\alpha$ as expected from the upper and lower surface pressures in the earlier figures, while the scaled drag gradually decreases. 

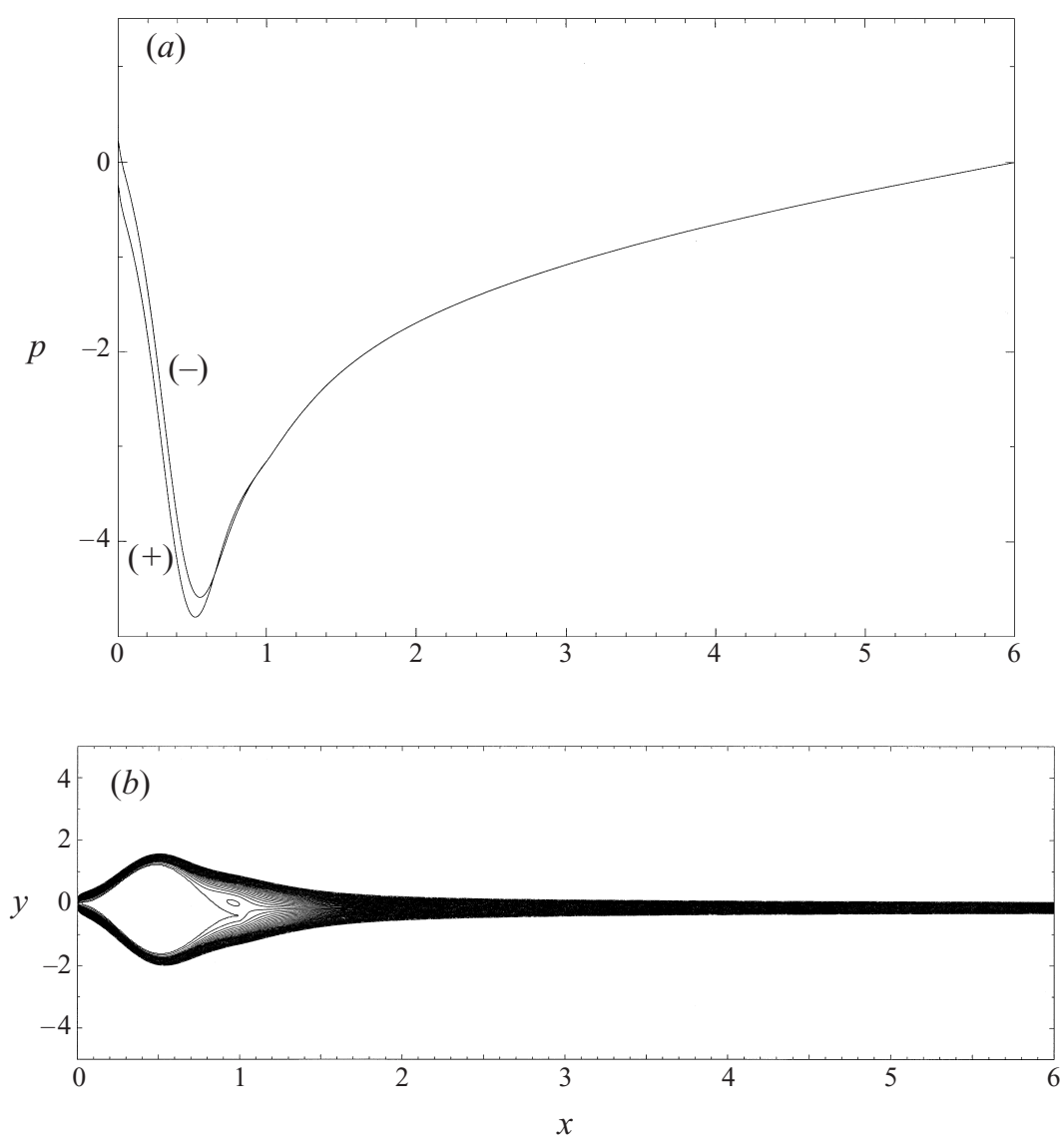

Figure 7. (a) Pressures and (b) close-up streamlines for the blade shape with $f^{*}=0.7$ at incidence $(\alpha=0.386)$, producing separated flow.

\section{Short-blade properties}

For short blades, the length $\ell$ is $r L$ with the ratio $r$ being small, and the spatial period $L$ from one blade leading edge to the next is now taken for convenience to be of order unity; we recall the generalization described in $\S 4.2$, such that $x$ in figures $3-10$ can be replaced by $x / \ell$ in effect. The orders of magnitude of the present scaled pressure, velocities and coordinates need to be considered here for small $r$ values.

There are then at least five subregions in the upper half of the non-symmetric flow solution of (2.2) and (2.3) even without separation taking place, as suggested in the symmetric case of Bowles \& Smith, accompanied by corresponding subregions in the lower half. Subregion $I^{ \pm}$is the viscous attached sublayer on the upper and lower surfaces of the blade and its immediate viscous wake, with streamwise length scale of order $r$ in $x$, and on the same streamwise length scale there is an inviscid subregion $I I^{ \pm}$outside and a passive inviscid sublayer $V^{ \pm}$in between them. The other two major subregions are of streamwise extent $O(1)$, namely $I I I^{ \pm}$, which is the continuation of subregion $I I^{ \pm}$, and $I V$, which at its upstream end is fed partly by the expanding wake that emerges downstream from sublayer $I^{ \pm}$and at its downstream end as $x$ approaches $L$ forms the beginning of subregion $V^{ \pm}$at the start of the period (next blade). The velocity profile of $V^{ \pm}$is in fact unaltered to leading order on the $O(r)$ streamwise scale and so that profile then constitutes part (the outer part) of the 

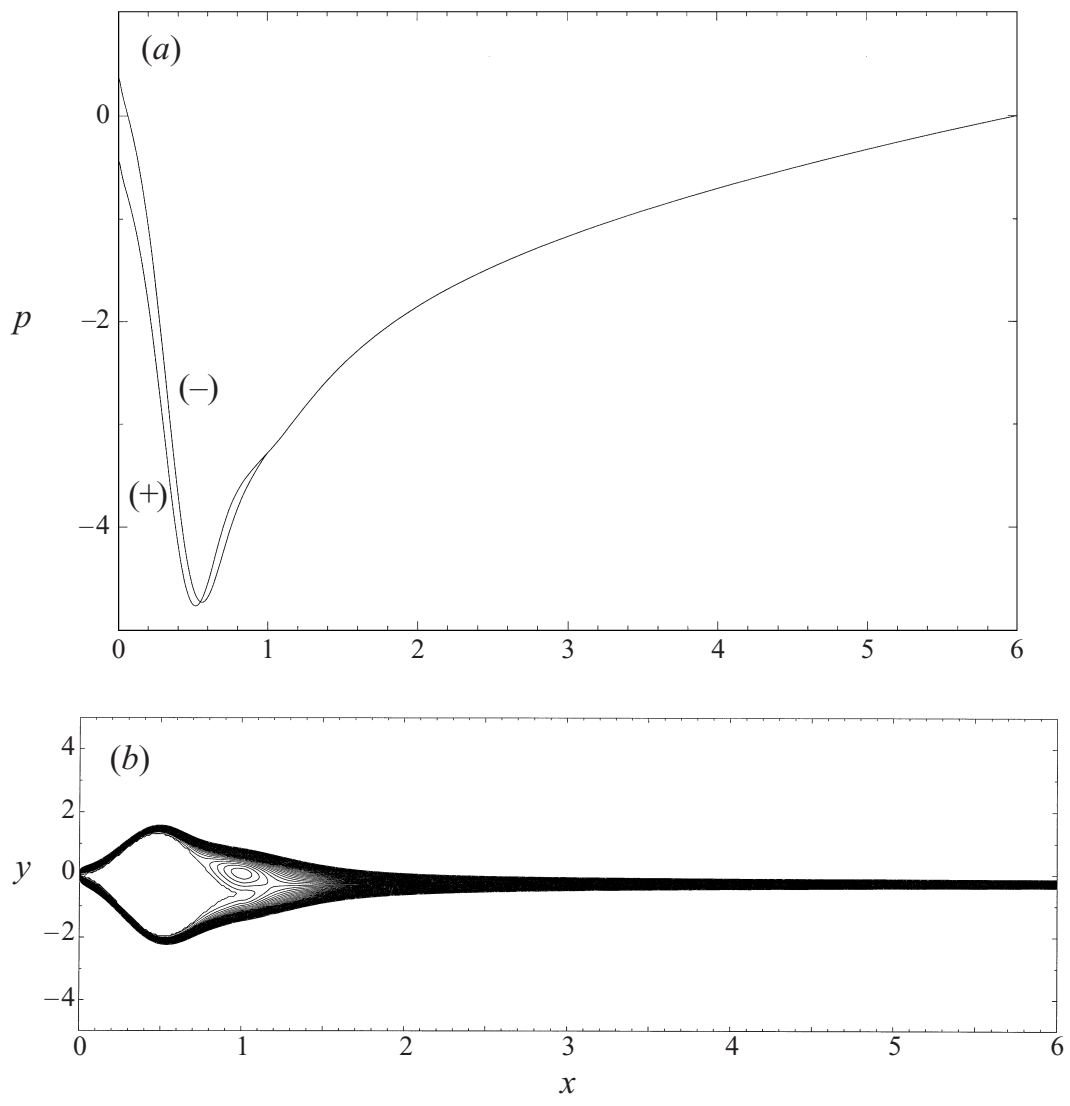

Figure 8. ( $a$ ) Pressure and (b) close-up streamlines for blade shape $f^{*}=0.8$, at scaled incidence $\alpha=0.575$, with separated flow.

starting profile for subregion $I V$ itself. See figure 11 which provides a sketch of the suggested flow structure and its subregions for small $r$ values.

\subsection{The main shorter length scale}

In the present non-symmetric context, the subregion IV remains largely symmetric as in Bowles \& Smith and the principal non-symmetric effects are felt instead in the short-scale subregions $I^{ \pm}, I I^{ \pm}$with smaller non-symmetric alterations also in the longer-scale subregions $I I I^{ \pm}, V^{ \pm}$.

The thickness of subregions $I I^{ \pm}, I I I^{ \pm}$is $O\left(r^{-1 / 3}\right)$ in $y$, from an order-of-magnitude argument, with a resultant velocity profile $u=r^{-1 / 3} U_{0}^{ \pm}(Y)$ which is unknown but independent of $x$, with $y=r^{-1 / 3} Y$. The profile $U_{0}^{ \pm}$tends to $\lambda^{ \pm}|Y|$ plus a constant at large $|Y|$ and is positive. The thinner sublayers $I V, V^{ \pm}$both have $O\left(r^{1 / 6}\right)$ thickness and sublayer $I^{ \pm}$is still thinner, with $\left|y-f^{ \pm}\right|$of order $r^{2 / 3}$. The characteristic blade widths $\left|f^{ \pm}\right|$here are supposed to be of order $r^{2 / 3}$ also and in consequence they appear directly in the upper and lower sections of the viscous layer $I^{ \pm}$on the blade, these sections being classical attached boundary layers driven forward by the uniform speed $u \sim r^{-1 / 3} \sigma$ just outside, where the constant $\sigma$ is $O(1)$ and positive, and then joining into the thin displaced wake beyond the trailing edge. Here $\sigma \equiv U_{0}^{ \pm}(0)$ is taken to be continuous across $Y=0$. 
Lifting multi-blade flows with interaction
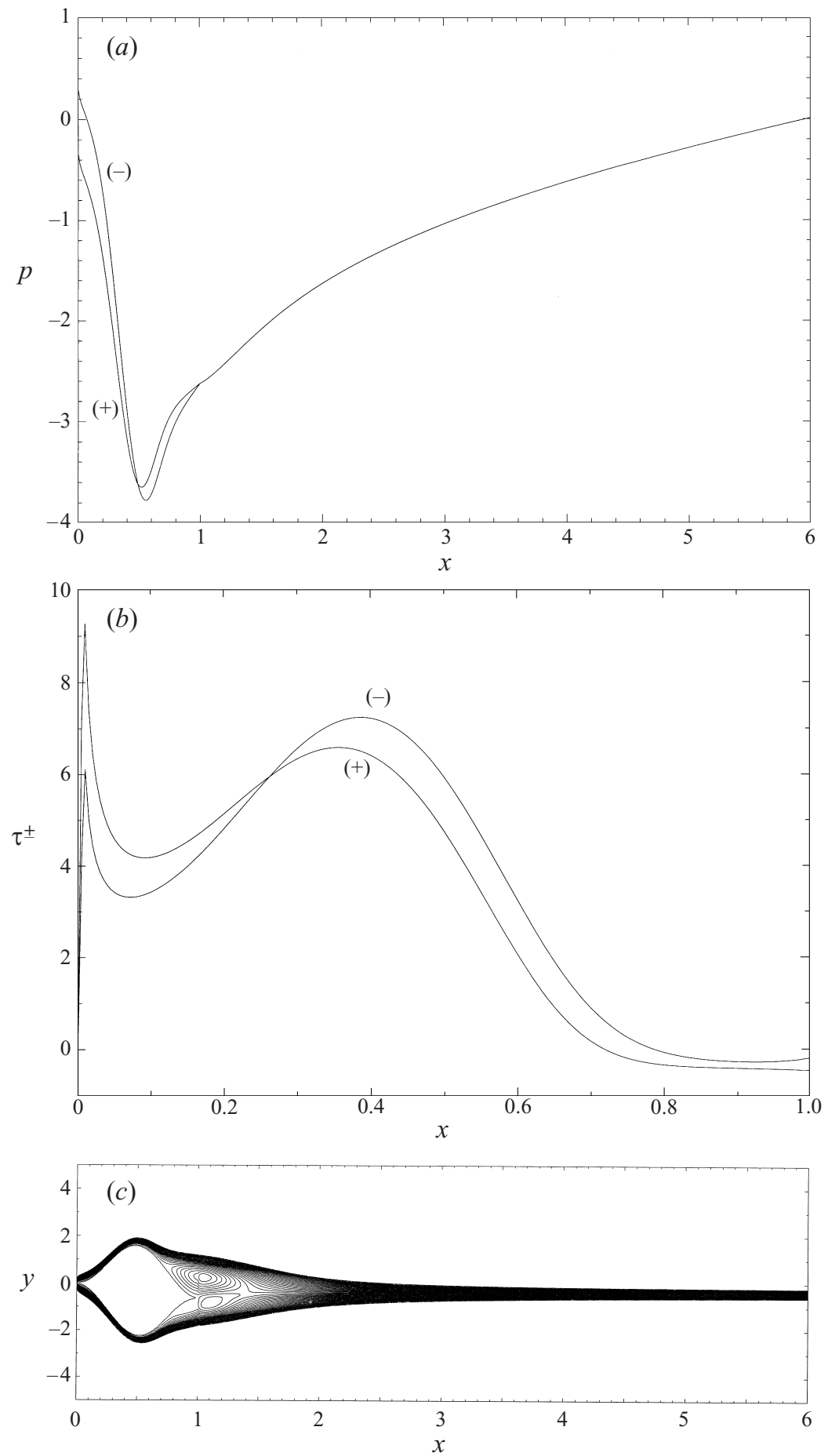

FIgURE 9. (a) Pressures and $(b)$ scaled skin frictions for blade shape $f^{*}=0.945$, incidence $\alpha=0.576$, with separated flow. (c) Streamlines. 


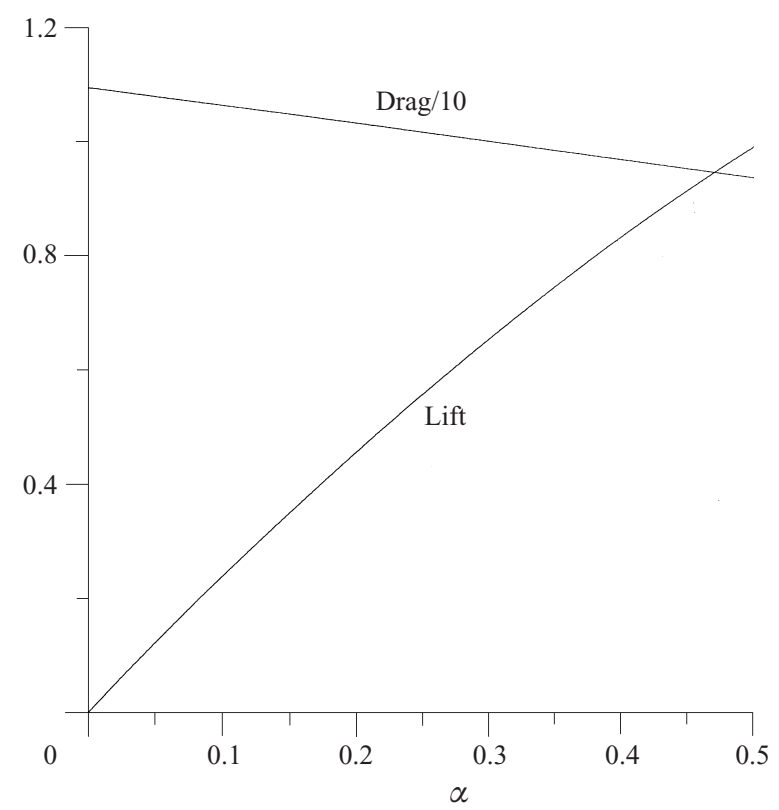

FIGURE 10. Scaled lift and drag versus scaled incidence angle $\alpha$, for flat-plate blades at incidence.
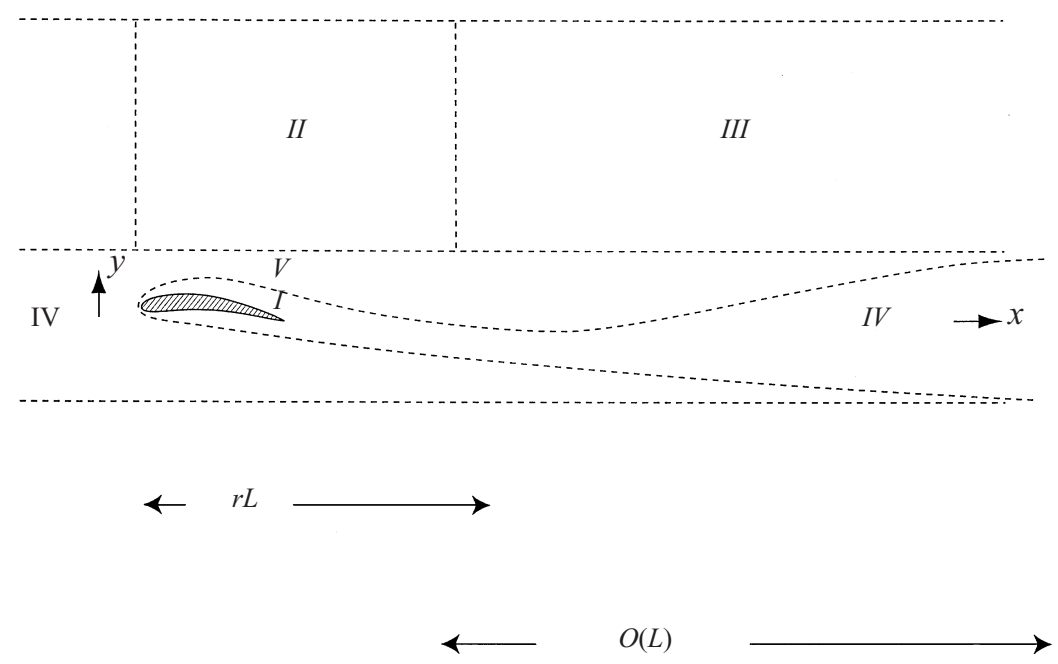

FIGURE 11. Flow regions induced when the blade lengths are short relative to the streamwise period; see $\S 5$.

The characteristic variation in the scaled pressure $p$ is of order $r^{1 / 3}$ over both of the $O(r), O(1)$ streamwise length scales, because of the relative thicknesses of $I, I I$ and the spatial periodicity; and within the former scale the inviscid balances in subregion $I I^{ \pm}$coupled with $(2.3 c)$ and matching at small $|Y|$ imply that

$$
p \sim r^{1 / 3}\left[\frac{-\delta(X) \mp f_{1,2}(X)}{J_{1,2}}+\pi_{1,2}\right],
$$

with the subscripts 1,2 now signifying values in the upper and lower halves respectively. Here $\delta$ is the scaled Blasius- and Goldstein-wake thickness, giving $\delta$ as $1.7208 \sigma^{-1 / 2} X^{1 / 2}$ 
on the blade and $\delta(\infty)=0.66412 \sigma^{-1 / 2}$; also $f_{1,2} \equiv f^{ \pm} / r^{2 / 3}$ is the scaled blade shape, impressed from sublayer $I$, with $x=r X$, while the constants $\pi_{1,2}$ are the unknown scaled pressure values at the leading edge. The other constants are the integrated quantities

$$
J_{1} \equiv \int_{0}^{\infty}\left(U_{0}(Y)\right)^{-2} \mathrm{~d} Y, \quad J_{2} \equiv \int_{-\infty}^{0}\left(U_{0}(Y)\right)^{-2} \mathrm{~d} Y,
$$

which are positive. In the thin wake section however, beyond the trailing edge, effectively $f_{1}=f_{2}$ is to be found from the equi-pressure condition $p^{+}=p^{-}$, giving

$$
\begin{aligned}
& f_{\text {wake }} \sim r^{2 / 3}\left[\frac{\left(J_{1}-J_{2}\right) \delta(X)+J_{1} J_{2}\left(\pi_{1}-\pi_{2}\right)}{\left(J_{1}+J_{2}\right)}\right], \\
& p_{\text {wake }} \sim r^{1 / 3}\left[\frac{-2 \delta(X)+\left(\pi_{1} J_{1}+\pi_{2} J_{2}\right)}{\left(J_{1}+J_{2}\right)}\right],
\end{aligned}
$$

from $(5.1 a, b)$. Also, to make the argument explicit, the leading-edge jump is taken as in (4.1), which requires the shift $y_{s}$ to be small, of order $r^{2 / 3}$ at most, say $y_{s}=r^{2 / 3} \bar{y}_{s}$, and gives the leading-edge pressure values as

$$
\pi_{1,2}=r^{-1 / 3} p(0-) \mp \lambda^{ \pm} \sigma \bar{y}_{s}
$$

to leading order, in terms of $\bar{y}_{s}$. On the other hand the trailing-edge constraint that $p^{ \pm}$(from $\left.(5.1 a, b)\right)$ must be continuous with $(5.2 b)$ at $X=1$ imposes the relation

$$
\pi_{1}-\pi_{2}=\left\{\left(J_{2}-J_{1}\right) \delta(1)+\left(J_{1}+J_{2}\right) f_{1}(1)\right\} /\left(J_{1} J_{2}\right),
$$

since $f_{1}=f_{2}$ at $X=1$; the same relation follows from requiring $f_{1,2}$ to be continuous with $f$ in $(5.2 a)$ at the trailing edge.

Hence the scaled shift $\bar{y}_{s}$ is determined, from (5.3) and (5.4), as the right-hand side of (5.4) divided by the negative quantity $-\left(\lambda^{+}+\lambda^{-}\right) \sigma$. If, for example, the magnitude of the blade deflection $f_{1}(1)$ is much greater than the viscous thickness $\delta(1)$ at the trailing edge then the sign of $\bar{y}_{s}$ is opposite to that of $f_{1}(1)$, meaning that a positive (negative) angle of blade incidence causes an upward (downward) displacement of flow just in front of the leading edge and a positive (negative) lift, which makes good physical sense.

With the integral properties $J_{1}, J_{2}$ assumed known it is interesting that the pressure distributions on the blade and in its immediate wake are determined to within an additive constant $(p(0-))$ by the local response of subregions $I, I I$, in (5.1)-(5.4), while the wake shape is determined fully, in (5.2a) with (5.4). On the other hand the pressure change over the $O(r)$ length scale from just ahead of the blade leading edge to the downstream end (far wake) of sublayer $I$ is given by $(5.2 b)$ evaluated at $X=\infty$ (where $\delta(\infty)$ is the Goldstein far-wake value), minus $p(0-)$, yielding the explicit result

$$
r^{1 / 3}\left[\frac{-2 \delta(\infty)+J_{2}\left(\pi_{2}-\pi_{1}\right)}{\left(J_{1}+J_{2}\right)}+\lambda^{+} \sigma \bar{y}_{s}\right]
$$

for this pressure change. Similarly the wake centreline position at the downstream end of sublayer $I$ is given explicitly by $(5.2 a)$, again with $X=\infty$, where $\pi_{1}-\pi_{2}$ is given in (5.4).

\subsection{The longer streamwise length scale}

The pressure change (5.5) has to be counter-balanced by an equal but opposite pressure change over the $O(1)$ streamwise length scale in order to satisfy the spatial 


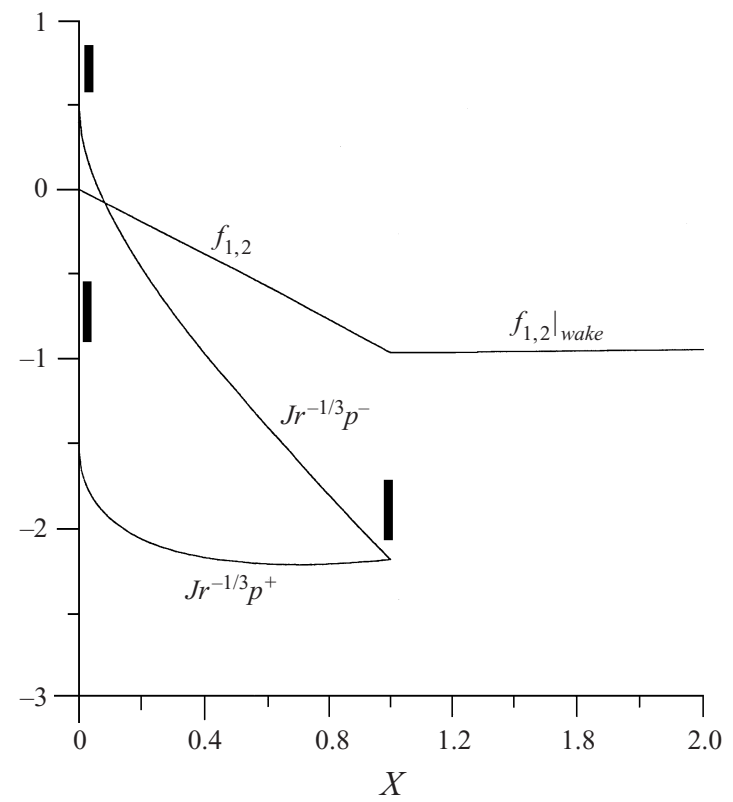

FIGURE 12. Scaled surface pressures and body and wake shapes, from the short-blade analysis, for unit values of $\sigma, \hat{\alpha}\left(=\alpha / r^{2 / 3}\right), \lambda^{ \pm}$, with $J r^{-1 / 3} p(0-)$ set at -0.5 and a blade which is an inclined flat plate. Here the case $J_{1}=J_{2}=J$ is taken. The three vertical bars indicate the range of pressure values obtained directly from figures $3-5$ for $\pi^{ \pm}$at the leading edge and $p$ at the trailing edge, for comparison.

$L$-periodicity condition. This is achieved through the flow responses in subregions $I I I, I V$, the former being similar to $I I$ except for an extra viscous contribution, so that

$$
p \sim r^{1 / 3}\left[\left(K_{1,2}+b\right) x / J_{1,2}\right]+\text { constant }
$$

on the $O(1)$ length scale, where $K_{1,2}$ denotes the integrals of $U_{0}^{\prime \prime} / U_{0}^{2}$ with respect to $Y$ from 0 to $\infty,-\infty$ to 0 respectively and the positive constant $b$ stems from the influx into the wake $I V$ as in Bowles \& Smith. Equating (5.6a) and (5.6b) imposes a constraint on the profile $U_{0}$ including the $c^{ \pm}$factors but more significantly the constant pressure gradient of (5.6) yields a pressure change of $r^{1 / 3}\left(K_{1}+b\right) L / J_{1}$ over this length scale. Hence we obtain, for periodicity of the pressure, the relation

$$
\begin{aligned}
\left(K_{1}+b\right) \frac{L}{J_{1}}=\frac{2 \delta(\infty)}{\left(J_{1}+J_{2}\right)}+\left\{\frac{J_{2}}{\left(J_{1}+J_{2}\right)}+\frac{\lambda^{+}}{\left(\lambda^{+}+\lambda^{-}\right)}\right\} & \\
& \left\{\left(\frac{1}{J_{1}}-\frac{1}{J_{2}}\right) \delta(1)+\left(\frac{1}{J_{1}}+\frac{1}{J_{2}}\right) f_{1}(1)\right\}
\end{aligned}
$$

linking together the long- and short-scale properties. The above assumes that, for the present $O\left(r^{2 / 3}\right)$ blade shape non-symmetries, the wake centreline is displaced in $y$ by an amount less than $O\left(r^{1 / 6}\right)$ ( $\mathrm{cf}$. (5.2a)), since otherwise the centreline shape would have to be linear in $x$ for consistency with (5.6) and then (5.3) would need altering, specifically through its $\sigma$ factor, and an unbounded stack of viscous wakes would be present in each spatial period. The relation (5.7) agrees with Bowles \& Smith's for symmetric flows. 


\subsection{An example}

An example of the pressure distributions and resulting wake shape from the shortblade theory is plotted in figure 12 , which takes the values $J_{1}, J_{2}$ to be equal, say to $J$, and unit values for $\sigma, \lambda^{ \pm}$and the scaled angle of incidence, with a flat-plate geometry. The pressure prediction shown in particular indicates fair agreement with the full results of $\S 4$ for quite moderate $r$ values, specifically with all the pressure curves in figures 3-5 where $r$ is $1 / 6$. Along with that, all the scaled skin friction or streamline displacement results in those figures are close to the Blasius forms predicted by the small-r analysis. This measure of agreement emerges despite the lack of knowledge of the $O(1)$ constants $J_{1}, J_{2}$, and so on, and suggests that the small-r theory may work well at even larger values of $r$ than $1 / 6$.

There is also agreement with the experiments and direct computations shown in Conlisk (1997, figure 8) whose form resembles quite closely that in our figure 12 and even shows signs of an underlying pressure jump at the blade leading edge. This is despite the different viscous thicknesses inherent in the present theory and in the above experiments or direct computations.

The drag remains essentially of the aligned flat-plate type in this regime. The scaled lift on the other hand is fixed to leading order by the short-scale response in $(5.1 a, b)$ ff. alone, as the lift relies on $p_{2}-p_{1}$ and hence on $\pi_{2}-\pi_{1}$ from $(5.1 a, b)$ (and on other quantities which are known), a pressure difference which is determined by the trailing-edge condition (5.4). For the example of flat-plate blades in figure 12, with equal $J_{1}, J_{2}$ and with $f_{1,2}$ equal to $-\hat{\alpha} X$ where $\hat{\alpha} \equiv \alpha / r^{2 / 3}$ is of order unity, we obtain the linear dependence

$$
\text { Lift } \sim\left(\alpha / J_{1}\right) r^{2 / 3}
$$

of the lift on incidence $\alpha$. This is in keeping with the computed behaviour of the lift for the $r$ value $1 / 6$ presented in figure 10 .

\section{Further comments}

\subsection{Immediate points}

We draw out three points immediately from the non-symmetric multi-blade flow study. First, the condensed periodic boundary layer and wake of $\S 2$, covering a wide parameter range, are as seen already in Bowles \& Smith's (2000) symmetric flow theory except for the streamwise jumps across the leading edge(s), described in detail in $\S 3$. These jumps are necessary to enable the condition of equal upper and lower surface pressures to be satisfied at the trailing edge of the blade. The jump mechanism involved, which appears to be new, balances the large shear-based inertial force against the large pressure gradient locally, inducing a jump in mass flux that is closely connected to the jump in pressure (and on a larger scale to the normal flow through the system). The mechanism here is different from that found at effective leading edges in branching flows and in vehicle-ground interference, because here normal interplay of pressure between two or more solid surfaces is absent.

Second, the short-blade analysis of $\S 5$ seems to capture the main physics of the numerical results at moderate chord/period ratios $(\S 4)$, at least for attached motions with moderate lift. One-sided or non-symmetric separations resembling those in Smith (1983) and Jones \& Smith (2000) tend to occur at higher lift or blade-thickness values in the results of $\S 4$ and can be incorporated in the short-blade theory as they are in Bowles \& Smith for non-lifting cases. In addition, the short-blade theory adds explicit 
support to the basic need for the leading-edge jump arising in virtually all lifting cases.

Third, the flow structure for blade-wake interaction with leading-edge jumps present extends to other contexts. It holds for example in the alternative setting of one isolated blade immersed non-symmetrically in the shear flow of a relatively thick wake generated upstream of the blade. Here the upper and lower pressures generated in the wake of the isolated blade must not only be equal but also tend far downstream to the undisturbed incident value, analogous with the conditions required in the current periodic flow setting. Applications that spring to mind are to successive blade row interactions in turbines, to trailing-edge flaps and to sheltering wake flight.

It is also interesting to consider briefly how the flow solution depends on the nondimensional blade chord length $\ell$ and streamwise period $L$, especially in the context of the applications described in the introduction. Suppose $\ell$ is fixed, within the range of (2.1). Then if $L$ is of order $\ell$, results qualitatively similar to those in $\S 4$ and figures 3-10 apply. If $L$ is much larger than $\ell$, however, the ratio $r$ is small, the blade is relatively short and so $\S 5$ applies. As $L$ continues to increase $\S 5$ continues to apply despite the thickening of the outer subregions until in effect the right-hand restriction in (2.1) is approached, for example through the definition of the characteristic length $\ell_{D}$ in $\S 2$. When that restriction is actually reached the only change to the theory in $\S 5$ is that a pressure-displacement linkage comes into play on the longer scale of $\S 5.2$, as in Bowles \& Smith (2000) for the symmetric case. If $L$ is reduced, by contrast, there is an intriguing limit case with the wake length $L-\ell$ becoming comparatively small, as discussed by Smith \& Timoshin $(1996 a, b)$. Next suppose $L$ is fixed and $\ell$ is varied instead. The maximum $\ell$ value corresponds to the case of small wake length just mentioned, while $\ell$ being of order $L$ is the subject of $\S \S 2-4$. Further reducing $\ell$, however, makes the ratio $r$ small and hence brings in $\S 5$ again. This continues to apply until, and possibly beyond, the stage where the characteristic streamline slopes of order $\delta_{1} r^{-4 / 3}$ in the outer subregions become of order unity, yielding a critical ratio $r$ of order $\left(R e^{-1} \delta\right)^{1 / 4}$ at which those subregions come under the control of the linearized or nonlinear Euler equations, depending on blade thickness. That critical ratio is small in view of the definition (2.1) for the range of current flows.

\subsection{Further studies and issues}

Some inkling of likely further studies of interest has been given already. Others are these. The special longer scale associated with the right-hand restriction of (2.1) can be addressed as in our previous study, bringing in direct pressure-displacement linkage either through the fully computational flow solution or on the longer length scale alone in the short-blade context as described above. Similarly, relatively thicker blades tend to bring into play the incident velocity profile of the whole boundary layer/wake rather than just the inner portion of uniform shear. Three-dimensional flows would undoubtedly be of interest, although computations with a trailing edge present are difficult. The short-blade theory may be more fruitful, starting with spanwise effects acting on the longer length scale. Unsteadiness likewise draws attention to the long scale first, for global features, but with the transition study of Smith et al. (2000) also being relevant in the near wakes and, along with other wake transition studies, reminding us of the turbulent wakes that occur in practice in some contexts.

Slightly different questions are raised by non-zero incidence of the entire multiblade system, rather than of individual blades relative to the system itself as in $\$ \S 4$ and 5. Suppose that the system incidence angle $\beta$ is small. Now the development length characteristic of the $O\left(\delta \ell_{D}\right)$ thick surrounding viscous motion in $\S 2$ is of 
order $\gamma \ell_{D}$ say, where $\gamma$ is large and given by $R e^{-1 / 2} \gamma^{1 / 2}=\delta$. So the system incidence provides a vertical shift in $y_{D}$ of order $\beta \gamma \ell_{D}$ at the typical blade downstream, whereas the viscous sublayer thickness in $y_{D}$ is $\delta_{1} \ell_{D}$ where $\delta_{1}$ is $\left(R e^{-1} \delta\right)^{1 / 3}$ from $\S 2$. The two $y_{D}$ estimates are thus comparable, inducing a first nonlinear effect of the system incidence, if $\beta \gamma=\delta_{1}$; that is, $\beta=O\left(R e^{-4 / 3} \delta^{-5 / 3}\right)$ determines the critical angle. Here $R e$ lies in the range (2.1). Hence $\beta$ must lie between the orders $R e^{-1}$ and $R e^{-3}$, or, which is equivalent, $\beta$ must be much less than both $\delta^{5}$ and $\delta^{-3}$. For instance if $\delta$ is about $\frac{1}{5}$ then the critical $\beta$ is approximately $8 \times 10^{-5}$, while if $\delta$ is about 5 the critical value is approximately $2 \times 10^{-3}$. These critical values of the angle $\beta$ are remarkably tiny. Moreover, on the local length scale of a blade and its wake the system incidence makes itself felt predominantly through the above vertical shift alone, not through a local incidence factor, since the typical local flow angle is of order $\delta_{1}$ which is much larger than the system incidence angle $\beta$. The flow structure of $\S \S 2$ and 3 therefore still holds here except that the no-slip constraint $(2.3 a)$ now applies with a constant $\bar{\beta}$ added to $f^{ \pm}(x)$, where $\bar{\beta}$ is the scaled angle of incidence of the entire system. The simple effective shift in the vertical coordinate locally again introduces non-symmetry into the periodic single blade-wake motion, representing the incidence of the multi-system.

Thanks are due to Susan Brown, Robert Bowles, Alan Jones and Sergei Timoshin for discussions and comment, to the referees for comments, to EPSRC and DERA Farnborough for support, and to the late Sir James Lighthill for a probing question on the flow structure.

\section{REFERENCES}

Bowles, R. G. A. \& Smith, F. T. 2000 Interactive flow past multiple blades and wakes. Q. J. Mech. Appl. Maths 52, 1-45.

Brouwer, H. H. 1992 On the use of the method of matched asymptotic expansions in propellor aeronautics and astronautics. J. Fluid Mech. 242, 117-144.

Conlisk, A. T. 1997 Modern helicopter aerodynamics. Ann. Rev. Fluid Mech. 21, 515-567.

Davis, S. S. \& Chang, I.-C. 1986 The critical role of computational fluid dynamics in rotary-wing aerodynamics. AIAA Paper 86-0336.

Egolf, T. A. \& Sparks, S. P. 1985 Hovering rotor airload prediction using a full-potential flow analysis with realistic wake geometry. In 41st Ann. Forum of the Am. Helicopter Soc., May 15-17, Fort Worth TX.

Hawkings, D. L. \& Lowson, M. V. 1974 Theory of open supersonic rotor noise. J. Sound Vib. 36, $1-20$.

Jones, M. A. \& Sмith, F. T. 2000 Fluid motion for car undertrays in ground effect. Phil. Trans. R. Soc. Lond. A (to appear).

LANDGRebe, A. J. 1994 New direction in rotorcraft computational aerodynamics research in the U.S. AGARD Rep. 1.

LORBER, P. F. 1990 Aerodynamic results of a pressure-instrumental model rotor test at the DNW. In 46th Ann. Forum of the Am. Helicopter Soc. May, Washington, DC.

Parry, A. B. \& Crighton, D. G. 1989 Asymptotic theory of propellor noise: I. Subsonic singlerotation propellor. AIAA J. 27, 1184-1190.

Seddon, J. 1990 Basic Helicopter Dynamics. Oxford: BSP Professional Books.

SмIтH, F. T. 1983 Interacting flow theory and trailing-edge separation-no stall. J. Fluid Mech. 131, 219-249.

Smith, F. T., Bowles, R. G. A. \& Li, L. 2000 Nonlinear effects in absolute and convective instabilities of a near-wake. Eur. J. Mech. B (in press).

Smith, F. T. \& Jones, M. A. 2000 One-to-few and one-to-many branching tube flows. J. Fluid Mech. (to appear). 
Smith, F. T. \& Timoshin, S. N. 1996 a Blade-wake interactions and rotary boundary layers. Proc. R. Soc. Lond. A 452, 1301-1329.

Smith, F. T. \& Timoshin, S. N. $1996 b$ Planar flows past thin multi-blade configurations. J. Fluid Mech. 324, 355-377.

Stewartson, K. 1960 A note on lifting line theory. Q. J. Mech. Appl. Maths 13, 49-56.

Strawn, R. C. \& Cardonna, F. X. 1986 Numerical modelling of rotor flows with a conservative form of the full-potential equations. AIAA Paper 86-0079.

WAKE, B. E. \& BAEDER, J. D. 1994 Evaluation of the TURNS analysis for hover performance prediction. In Am. Helicopter Soc. Aeromech. Specialists Conf., 19-21 January, San Francisco, $C A$ 